\title{
Les femmes et les enfants, prisonniers politiques ou déportés à titre préventif pendant la guerre civile grecque
}

\section{Christina Alexopoulos}

\section{CpenEdition}

\section{Journals}

Édition électronique

URL : https://journals.openedition.org/ceb/830

DOI : $10.4000 /$ ceb.830

ISSN : 2261-4184

Éditeur

INALCO

\section{Édition imprimée}

Date de publication : 30 mars 2011

Pagination : 267-288

ISBN : 978-2-85831-189-7

ISSN : 0290-7402

\section{Référence électronique}

Christina Alexopoulos, «Les femmes et les enfants, prisonniers politiques ou déportés à titre préventif pendant la guerre civile grecque », Cahiers balkaniques [En ligne], 38-39 | 2011, mis en ligne le 06 décembre 2011, consulté le 06 juillet 2021. URL : http://journals.openedition.org/ceb/830 ; DOI : https://doi.org/10.4000/ceb.830

Ce document a été généré automatiquement le 6 juillet 2021.

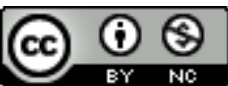

Cahiers balkaniques est mis à disposition selon les termes de la Licence Creative Commons Attribution - Pas d'Utilisation Commerciale 4.0 International. 


\title{
Les femmes et les enfants, prisonniers politiques ou déportés à titre préventif pendant la guerre civile grecque
}

\author{
Christina Alexopoulos
}

Pendant la guerre civile grecque (1946-1949) des milliers de personnes sont emprisonnées, déportées dans des camps de concentration ou exilées dans des îles. À l'intérieur du groupe des prisonniers politiques se trouvent différentes catégories de détenus, formant une population hétéroclite d'hommes et de femmes, persécutés pour leurs idées, leurs origines ou même des liens de parenté avec les communistes. Dans la présente étude, nous nous intéresserons à un groupe sociologique particulier, lui-même assez peu homogène, les femmes et les enfants, prisonniers politiques ou déportés à titre préventif pendant le conflit. Après un bref rappel de la pratique de la déportation dans la guerre civile et une esquisse de typologie de la réclusion, nous nous attacherons à étudier les conditions de détention et les stratégies de survie des femmes, souvent emprisonnées avec leurs enfants, leur statut au sein de la communauté des prisonniers politiques ainsi que le sens de l'engagement politique dans leur système de valeurs.

\section{La pratique de la déportation et de l'emprisonnement dans le contexte politique de la guerre civile grecque.}

\section{Rappel historique.}

2 Dans le contexte politique de la guerre civile grecque, la déportation et l'emprisonnement servent de moyens pour intimider une population considérée comme susceptible de participer à l'effort de guerre en apportant une aide matérielle ou morale aux combattants communistes. Comme les récents travaux de Polyméris Voglis ${ }^{1}$ l'ont démontré, l'arbitraire des arrestations, la durée de la détention avant le 
procès ou à titre préventif, la sévérité des peines prononcées pour des "délits » spécialement inventés voire non commis tout comme l'arsenal juridique créé ad hoc ${ }^{2}$ pour contrer «la menace communiste " participent, au même titre que les exécutions sommaires, la torture et les exactions des groupes paramilitaires d'extrême droite, d'une politique d'exclusion violente de la gauche où le maître mot est la terreur. Coupable de sa participation au projet ${ }^{3}$ de renouveau de l'EAM pendant la résistance, suspectée d'adhérer aux idées communistes ou ayant juste la mauvaise idée de se trouver au mauvais endroit au mauvais moment, la partie de la population grecque qui pourrait être amenée à collaborer avec l'Armée démocratique doit être intimidée, tout comme les rebelles qui doivent se couper de leur milieu naturel.

3 Cette situation conduit à des déplacements de population depuis les zones rurales vers la périphérie des grandes villes, entrâne la déportation sur des îles d'exil des femmes et des enfants ayant un lien idéologique ou un lien de parenté avec les communistes (eux-mêmes combattants, prisonniers politiques ou rentrés en clandestinité) et impose le départ au bloc de l'Est de milliers d'enfants, nés de parents communistes, qui n'ont guère d'autre alternative que de quitter le pays, pour fuir les représailles des paramilitaires et l'envoi forcé dans des orphelinats et autres camps disciplinaires d'endoctrinement anticommuniste créés par la reine Frédérica. ${ }^{4}$

4 Sur les lieux d'exil et d'enfermement se retrouve alors une population hétéroclite comportant entre autres des femmes déportées à titre préventif accompagnées d'enfants en bas âge, des combattantes inculpées pour leur participation au conflit, des membres du Parti communiste, des personnes rentrées en clandestinité pour fuir les représailles des paramilitaires, voire grand nombre de femmes âgées, dont les fils ou les filles sont au maquis.

\section{Présentation des principaux camps et prisons}

5 Ces femmes se retrouvent dans différents camps de déportations et prisons, après un passage par les locaux de la Sûreté et autres postes de police, où l'usage de la torture est quasi systématique. Nous allons évoquer quatre camps d'enfermement et de déportation relatés dans les récits d'anciens prisonniers politiques, à savoir les camps de Chios, de Trikeri, de Makronissos et de Aï Stratis, ainsi qu'une prison pour femmes située à Athènes, la prison Avéroff, puis nous essaierons d'esquisser une typologie des espaces de réclusion.

6 En mars 1948, 94 femmes et 17 enfants sont déportés à Chios, suivis d'un deuxième groupe de 75 femmes et de 13 enfants, puis d'un troisième groupe de 58 femmes et 3 enfants. Très rapidement, on passe à des envois de 50 à 100 personnes deux fois par semaine. Condamnés officiellement à une simple déportation sans enfermement, ces détenus se voient très rapidement subir une double peine celle de l'enfermement dans des bâtiments surpeuplés et insalubres, puis une nouvelle peine vient s'y rajouter, sous forme de privations : manque de nourriture, d'eau, de soins médicaux, interdiction de correspondance, violences physiques et psychologiques, divisions de femmes en plusieurs groupes de "dangerosité » avec institution d'une "Annexe spéciale », de juillet 1948 en avril 1949.

7 En avril 1949, les détenus du camp de Chios sont transférés au camp de Trikeri, une île non habitée en Thessalie. Dans ce camp se trouvait déjà depuis 1947, le campement d'hommes qui comptait trois mille à quatre mille déportés, transférés en 1949 à 
Makronissos. Dès 1947, on y pratiquait la déportation à titre préventif des femmes qui avaient un lien de parenté avec les communistes et qui se trouvaient dans des zones rurales investies par l'armée gouvernementale. Leur nombre n'était pas stable ${ }^{5}$, en 1948, il y en avait 500 en février 1949. (Le seul moyen de quitter le camp, était de signer une déclaration de repentir, abjurant sa famille et condamnant comme traîtres les parents communistes). Dans ce camp arrivent donc en 1949, 1200 détenus du camp de Chios dont 150 enfants et à peu près 200 femmes très âgées et, après l'arrivée des Slavomacédoniennes et des Épirotes, le nombre de femmes et enfants emprisonnés passe à 3500 personnes. En septembre 1949, ce nombre, sur place, dépasse les 4700 personnes. Parmi ces femmes, il y a également des combattantes de l'armée démocratique, qui sont enfermées dans un camp à part. Dans la population déplacée, il y beaucoup de malades, atteintes de tuberculose, de typhus et de malaria et quelques cas de nouveau-nés qui meurent faute de soins. ${ }^{6}$ Plusieurs se voient obligées de signer la déclaration de repentir dans l'espoir de sauver leurs enfants ${ }^{7}$, des enfants qui subissent au même titre que les adultes des peines disciplinaires. ${ }^{8}$. En janvier 1950, de nombreuses femmes déportées à titre préventif, finissent par signer la déclaration de repentir. La guerre civile est finie sur le plan militaire depuis le 29 août 1949, les membres de leurs familles, engagés du côté démocratique, sont tués ou faits prisonniers, leurs enfants en bas âge sont à l'abandon quand ils ne sont pas avec elles dans le camp.

Pour celles qui refusent de signer, le transfert à Makronissos en janvier 1950 est vécu dans une grande appréhension, la réputation de ce camp de rééducation et d'expérimentation de nouvelles tortures n'est plus à faire, les prisonnières savent pertinemment en quittant Trikeri, ce qui les attend là-bas. Arrivées à Makronissos, et après confrontation avec des "repentis ", qui leur racontent les supplices qu'ils ont subis avant de signer la déclaration de repentir, les femmes doivent se séparer de leurs enfants. De nouvelles tortures visant à leur extorquer la déclaration ont lieu pendant plusieurs jours, en fin de compte plus de la moitié de ces femmes refuse de signer et est renvoyée en août 1950 à Trikeri, d'où ces femmes ne partiront qu'en 1953 pour le camp de Aï Stratis.

9 Après ce descriptif des camps, une référence rapide aux prisons et orphelinats s'impose. Dans les prisons de femmes tout comme dans les prisons d'hommes, les conditions de détention sont très difficiles, la surpopulation carcérale, l'absence d'hygiène, le manque de nourriture, le sadisme de certains gardiens et la confrontation avec des expériences extrêmes, comme les exécutions ou la torture, rendent le quotidien des détenus difficile à supporter. Il existe néanmoins une certaine disparité dans les conditions de détention en ville et en campagne. Les prisons en zone rurale sont davantage exposées à la Terreur Blanche et autres actes de barbarie perpétrés par les groupes paramilitaires d'extrême droite. Partout, les conditions sont très difficiles pour les enfants, qui n'existent pas pour l'administration carcérale, et n'ont donc pas droit à des rations alimentaires.

10 Prenons l'exemple de la prison pour femmes Avéroff. L'arrivée à la prison est un soulagement pour les femmes, spécialement celles qui ont des enfants, les conditions de détention y sont nettement meilleures que celles des commissariats, locaux de sûreté et autres espaces réservés aux interrogatoires, même si le manque d'hygiène, d'eau potable et de nourriture, mêlé à la promiscuité rendent leur quotidien très difficile. Les mères, qui sont toujours aidées par des camarades, gardent avec elles leurs enfants jusqu'à ce qu'ils atteignent l'âge de deux ans, puis les enfants doivent quitter la prison, 
dans le meilleur des cas pour être pris en charge par de la famille ou des voisins dans le pire des cas pour être placés en institution. À partir du printemps 1948, 14000 enfants sont placés en institution, tandis qu'à peu près 25000 traversent la frontière dans le nord du pays et sont placés dans des Démocraties populaires. ${ }^{9}$

En 1949, la prison de femmes Avéroff compte dix fois plus de prisonnières qu'elle ne peut en accueillir ${ }^{10}$, la majorité des femmes ont entre 20 et 40 ans, la plus jeune a 12 ans, la plus âgée 80. Dans la période 1945-1950, 106 mères et 119 enfants vivotent dans cette prison, l'espace imparti à une mère et ses deux enfants ne pouvant excéder les deux mètres carrés. ${ }^{11}$ En août 1950, la direction de la prison ordonne le départ des enfants, âgés de plus de deux ans (93 enfants), afin de punir les femmes de leur mouvement de protestation contre l'exécution de prisonniers politiques. 37 enfants aboutissent dans la villa Katsigéra à Kefalari, une propriété confisquée par les autorités et transformée en orphelinat, où les enfants manquent de tout (literie, vêtements, meubles, etc.). Puis au printemps 1952, tous les enfants sont transférés au village/ sanatorium de Kalamaki où les conditions sont un peu meilleures. De manière générale, ces orphelinats observent une discipline militaire, pratiquent largement les châtiments corporels et isolent les enfants de leur entourage familial (communication quasi inexistante.) Leur mode de fonctionnement n'est pas éloigné de celui des institutions pénitentiaires et l'embrigadement idéologique continue à jouer un rôle très important ; il s'agit toujours de rééduquer ces "enfants de traîtres ». En prison et en camp avec leurs mères, ou seuls en orphelinat, les enfants doivent toujours gérer des situations de précarité et d'incertitude constantes, et sont utilisés par les pouvoirs publics comme un moyen de pression supplémentaire pour l'obtention de la déclaration de repentir.

Les enfants partis à l'Est, globalement mieux reçus par les gouvernements en place (parfois même chouchoutés par le régime et assimilés à des pupilles de la nation) ont globalement connu un sort plus heureux ${ }^{12}$.

\section{Une typologie des espaces de réclusion chez les femmes prisonnières politiques ou déportées à titre préventif.}

13 Si nous essayons d'esquisser une typologie de la réclusion des femmes, prisonnières politiques ou déportées à titre préventif en Grèce, en suivant en cela les travaux de P. Panayotopoulos, nous pourrons aboutir à l'élaboration de deux classifications. Une première classification selon le caractère plus ou moins ouvert ou fermé de l'espace de déportation, ce qui influe sur les possibilités d'autogestion que les détenus peuvent avoir, et une seconde en fonction de l'intensité de l'expérience traumatisante (sévices corporels, isolements, pratiques dégradantes, assassinats).

Dans des cas d'expérience extrême en lieu de réclusion fermée, comme dans le camp de Makronissos, ou comme dans les locaux de la sûreté où l'on pratique systématiquement la torture, les conditions de détention ne permettent pas aux prisonniers d'élaborer un mode d'organisation communautaire aussi étendu que celui qui est de vigueur dans des expériences moins extrêmes ou en réclusion ouverte. Dans les locaux de la sûreté, chacun est confronté à ses propres limites face à la douleur, on entend les cris de ceux qui se font torturer, mais on n'y peut rien. En prison, on est soutenu par la communauté et les témoignages nous parlent de ce bonheur de se sentir à nouveau « appartenir à l'humanité.$^{13}$ 
Pour apporter un bémol à cette première classification, réclusion ouverte versus réclusion fermée/expérience forte versus expérience extrême, il faudrait insister sur la fluctuation fréquente entre les différentes formes de réclusion dans un laps de temps assez limité en fonction des conditions politiques extérieures; d'où le besoin de se représenter le monde de la prison non pas de manière statique ou immuable, mais comme un régime dépendant des réalités extérieures et tributaire de l'évolution de la situation internationale et des objectifs du pouvoir en place.

Sur un axe historique, on peut affirmer que l'évolution est manifeste tant au niveau des pratiques coercitives qu'au niveau du but politique recherché. Avant la dictature de Métaxas de 1925 à 1936, la réclusion des communistes est de type ouvert, le gouvernement cherchant avant tout à construire l'image d'un ennemi de la société, à exclure du corps social par la pratique de la déportation. Le but recherché est aussi d'assimiler les communistes à des détenus de droit commun, ce dont ils se défendent en essayant de s'en démarquer. Pendant la dictature de Métaxas, l'image « d'ennemis de l'ordre social » commence à être bien implantée, le régime pratique la réclusion fermée et applique des mesures coercitives qui font des conditions de détention une expérience traumatique forte (Akronauplie) ou extrême (Corfou). La période de la guerre civile est dominée par la réclusion fermée généralisée, les communistes ne sont plus présentés comme des délinquants, mais comme des traitres à la Nation. Les femmes communistes sont aussi considérées comme ennemies de la Nation, mais cette formulation étant trop sobre, on préfère les traiter de "putes bulgares ", ce qui est autrement plus imagé. Entre 1955 et 1967, années de libéralisation progressive du régime anticommuniste, les réclusions ouvertes, moins onéreuses pour l'État grec, prennent à nouveau le dessus, enfin, pendant la dictature des colonels, la dichotomie entre camps d'exil (réclusion ouverte) et prisons (réclusion fermée) regagne en actualité.

17 Après cette tentative d'analyse typologique du système de réclusion dans ses différentes structures et périodes historiques, il serait intéressant de voir comment les femmes déportées ou emprisonnées organisent leur vie en prison et se défendent de la stigmatisation dont elles font l'objet, l'enjeu de l'image de soi étant primordial tant pour leur survie psychologique que pour leur lutte politique.

\section{Les stratégies de survie}

18 Nous allons présenter les stratégies de survie des prisonnières politiques, leurs pratiques de la résistance, mais aussi la mise en place d'une infrastructure parallèle, celle du bureau du parti qui dédouble l'appareil carcéral.

\section{Existe-t-il des pratiques de résistance intracarcérale spécifiques aux femmes?}

19 À la différence des hommes pour qui l'image du combattant vertueux, tout dévoué à sa cause, semble incompatible avec l'autodérision ou l'humour, les femmes ont recours à la parodie, la mise en scène d'un quotidien pesant, et l'introduction décisive de certaines pratiques corporelles subjectivantes. 
Comme l'explique si bien Panayotopoulos ${ }^{14}$, « il s'agit avant tout de rire, de faire de l'humour, ${ }^{15}$ de créer les conditions d'une "évasion", d'introduire la distraction, dans toutes ses significations. Comportements que les hommes communistes cherchent généralement à limiter. Humour qui se conjugue avec une théâtralisation du discours et une mimétisation autosarcastique des actes de la vie quotidienne. ${ }^{16}$ Le théâtre, sous toutes ses formes ${ }^{17}$ est à notre avis le support de ces méthodes de résistance singulières.$^{18}$ Les jeux de mots sont un autre exercice de résistance récurrent en tant que figure d'humour emblématique de la déconstruction du discours et de la négation des rôles socialement assignés par le langage. ${ }^{19}$ L'ensemble de ces pratiques n'élargit pas seulement l'espace subjectif des recluses par son caractère solidaire et humanisant, il contribue à resocialiser cette population mise en marge des réseaux sociaux ordinaires. Nous pouvons citer l'exemple impressionnant de l'installation d'un salon de coiffure sur l'île de Youra, confectionné et organisé par les recluses elles-mêmes, afin de pouvoir s'embellir et se "remonter le moral"20. L'absence d'objets de désir, que nous pourrions assimiler à l'absence d'hommes pourrait donner à cette entreprise un caractère ridicule ; à notre avis c'est justement cette absence qui lui rend un intérêt particulier, puisque l'embellissement devient un besoin en soi, un signe de l'autopoïèse subjective des recluses. [...] Il ne faudrait pas pour autant, s'enchanter à l'idée d'avoir enfin trouvé une pratique alternative à ces pratiques unifiant la résistance dans un discours idéologique, celui du parti. La coupure que nous constatons s'inscrit dans l'absence de la "dimension politique pleine" accordée aux femmes. Nous soutenons par là que la facilité avec laquelle les femmes organisent leur vie sur des bases différentes à celle des hommes provient en grande partie de leur assignation à un régime politique inférieur. [...] Si les femmes arrivent à dépasser les tabous en vigueur dans les schèmes de la morale combattante, si elles arrivent à "faire du cinéma", c'est bien qu'il y a une ouverture à cet "impudique libérateur" et pourtant, significatif du manque de poids des femmes ${ }^{21}$ dans la balance du stigmate social. Les hommes n'entrent jamais dans une logique d'autodérision de peur d'altérer l'image du héros intègre, sérieux et décidé à accomplir son devoir, de peur d'altérer la pureté de sa représentation masculine combattante.

21 Si nous nous plaçons, maintenant, d'un point de vue permettant d'examiner la question sous l'angle des pratiques discursives moralisantes instituées par le discours dominant dans le parti, nous voyons que les sujets à moraliser, à dresser pour parler en termes foucaldiens, sont une fois de plus les sujets masculins. Les femmes arrivent à développer des pratiques subjectivantes, en raison de la liberté, que leur position inférieure dans les grilles de la hiérarchie combattante du parti leur permet d'entretenir. Le parti s'applique à éduquer, à moraliser une catégorie d'élus : les acteurs politiques, les hommes.»

\section{Les équipes de survie.}

Les prisonniers politiques, et cela n'est pas spécifique aux femmes, tentent de s'organiser en équipes de survie pour mieux faire face à l'oppression carcérale. Cela leur donne la possibilité non seulement de mieux résister à la pression gouvernementale, mais aussi de transformer la prison en un espace privilégié de réalisation de leur rêve sociétal. Il est d'ailleurs révélateur que dans cette organisation d'inspiration communiste du quotidien, se retrouvent trait pour trait les aspects 
fondamentaux de la théorie et de la pratique communiste : il y a partage obligatoire et... surveillance généralisée.

Au départ, les équipes de survie sont constituées sur un mode pratique, on forme un groupe de femmes avec celles qui reçoivent des visites et donc des denrées alimentaires et celles qui, pour des raisons d'éloignement géographique, ne peuvent pas en avoir, l'objectif étant que tout le monde mange à sa faim. L'entraide est de règle, des cours d'alphabétisation ou d'enseignement général sont proposés, en fonction des compétences de ceux qui donnent et de ceux qui reçoivent les leçons. Le tout est chapeauté par un noyau dirigeant, qui est censé protéger l'unité du groupe de tout danger extérieur ou intérieur (risques d'entrisme, de déstructuration, d'infiltration et de dislocation ${ }^{22}$ ) et aider chacun des prisonniers à mieux résister ${ }^{23}$, l'idée sous-jacente étant que l'on résiste mieux en groupe dirigé qu'individuellement. Concrètement, cette structure vient dédoubler l'appareil carcéral, imposer des sanctions aux membres dissidents ou indisciplinés (allant jusqu'à l'exclusion de l'aile des prisonniers politiques ou l'isolement ${ }^{24}$ ) et classer des prisonniers politiques entièrement dévoués à leur cause à des catégories de fiabilité (il y en 5).

Les communistes grecs dans leur rapport à la discipline (superposition de deux règlements très stricts celui de la prison et celui du parti) dans le choix de leurs moyens de résistance (grève de la faim jusqu'à ce que mort s'ensuive, mais interdiction de l'évasion ou de la mutinerie), dans l'image qu'ils souhaitent donner d'eux-mêmes (morale ascétique, détachement du monde, absence d'affect ou de besoin charnel ${ }^{25}$ et sens du sacrifice ${ }^{26}$ ) sont pris dans la logique de leurs adversaires. Nous entendons par là qu'ils sont incapables de s'extraire du discours dominant et qu'à force de vouloir montrer leur non dangerosité sociale, et d'essayer de se constituer en exemple à imiter, en avant-garde de la révolution et de l'homme nouveau, pour la société grecque et son extension kantienne en société universelle, ils se conforment aux valeurs traditionalistes de leurs opposants (famille, travail et patrie). Autrement dit, il y a une surenchère dans l'expression du sentiment patriotique, ou de l'attachement à la famille qui n'est pas faite pour œuvrer dans le sens d'une plus grande liberté individuelle.

Le besoin de démarcation des détenus de droit commun conduit à la diabolisation de ces derniers, perçus comme du lumpenprolétariat (selon la formulation léniniste) et pour les femmes s'accompagne d'un besoin d'affirmation de leur "respectabilité sociale. » La sexualité en tant qu'expression privilégiée de la subjectivité individuelle est censurée, la question du désir est éludée, la femme communiste doit se distinguer par sa « moralité. » Il s'agit de reconquérir l'honneur bafoué dans une incessante quête de légitimation, au détriment d'une pratique plus radicale de la résistance intracarcérale. Au détriment aussi, de la reconnaissance de cette souffrance des corps et des âmes qu'impose l'idéal du communiste modèle, comme tout autre idéal d'ailleurs. La grève de la faim, qui unifie le sujet et l'objet de la violence dans le corps du reclus, et l'injonction de préférer la mort sous la torture à la déclaration de repentir, sont deux exemples probants de l'infléchissement de la valeur « vie humaine » vers la projection imaginaire d'une conduite idéalisée. Le corps des détenus, et cela est particulièrement vrai pendant la torture et les viols collectifs, devient le vecteur de la résistance collective à l'injonction de trahir. Tout se passe comme si la victime de la violence se détachait de sa corporéité, comme si finalement elle ne lui appartenait plus. qui ne peut s'exprimer qu'à travers une mémoire fusionnelle où le « je » n'existe qu'à 
travers le «nous ». La concordance des premiers récits testimoniaux, où le lecteur a l'impression de relire toujours la même histoire, est là pour nous dire que finalement pour ces femmes, la notion de subjectivité se place au-delà de la perception individuelle, dans une vision transcendante de l'engagement politique.

\section{Le sens de l'engagement politique}

Pour comprendre l'abnégation de ces femmes et leur capacité à résister, il est important d'interroger le sens que prend l'engagement politique pour elles, ce qui est loin de se résumer à leur perception de la chose publique. Quel est le parcours de ces femmes? Comment perçoivent-elles leur engagement? Quelle est leur place dans la mémoire de la guerre civile et dans l'écriture testimoniale?

Parmi elles, on compte aussi bien des membres de l'organisation de résistance EAM ou même du PC, des femmes conscientes donc de leurs choix idéologiques que des personnes soupçonnées (pour des raisons familiales ou personnelles) de sympathie à l'égard des communistes. Ce qui est intéressant, c'est la diversité sociologique des personnes déportées ou emprisonnées. Il s'agit de femmes de tout âge et milieu social, venant aussi bien des zones rurales que des centres urbains, et qui, pour ainsi dire, n'ont en commun que leur adhésion à une idéologie ou des valeurs jugées subversives. Cette diversité sociologique, mise en avant dans les récits testimoniaux, est moins présentée comme une explication de certaines difficultés « du vivre ensemble » dans le quotidien et plus comme une illustration, du caractère massif de la persécution de la gauche, et par là même, de l'adhésion tout aussi massive que l'EAM avait su susciter.

L'habitus de l'EAM pour reprendre l'analyse bourdieusienne de Riki Van Boeschoten, a eu une portée fédératrice transcendante à l'égard des différences d'âge, de milieu social ou de provenance régionale et dans ce sens, il a permis d'incarner un projet de société et un mouvement de changement novateurs qualifiés par les auteurs de gauche de « $\pi \alpha \lambda \lambda \alpha i ̈ \kappa \alpha ́$ » (qui embrasseraient toute la population.) Il a aussi permis pour la première fois aux femmes grecques, notamment en milieu rural, de prendre conscience de leurs droits et d'en obtenir du moins en principe une première reconnaissance. Leur lutte sociale s'est ainsi doublée d'une lutte identitaire, d'un combat pour la libération de la femme et ce serait peut-être là un élément d'explication supplémentaire de cet engagement quasi total, que le seul sens de l'honneur, si important soit-il dans la société rurale de la Grèce des années 40 , ne suffirait pas à expliquer. L'expérience de la résistance a concrètement changé leur quotidien, elles ont été investies d'une mission sociale, elles ont eu accès à la chose publique, elles se sont enfin senties autorisées à prendre la parole et à agir. Tout comme les minorités réprimées du Nord de la Grèce, les femmes de la résistance ont expérimenté une nouvelle forme de liberté. Une première explication de leur adhésion idéologique est donc à chercher du côté de l'expérience novatrice et libératrice de la résistance.

30 Pour expliquer les différentes composantes de l'engagement des femmes, il faudra également évoquer une série de valeurs, centrales dans leur système éthique, telles que la loyauté, la persévérance et la dignité. Face à l'épreuve de la torture, ce qui permet à ces femmes de tenir, ce n'est ni leur force physique, ni la connaissance théorique de la doctrine marxiste, c'est avant tout le sentiment de ne pas avoir le droit de faire autrement; de devoir conserver sa dignité et sa liberté de penser quel qu'en soit le coût. Les exemples des détenus non communistes qui refusent par sentiment de dignité 
de signer la déclaration d'abjuration montrent qu'au-delà de l'adhésion idéologique, l'enjeu premier, c'est bien celui du refus de la compromission. On s'attendrait à des arguments idéologiques, or même une grande intellectuelle comme Rosa Imvrioti déjà très âgée au moment où elle se fait torturer et qui craint de ne pas tenir, n'évoque pas d'arguments intellectuels pour expliquer son refus de céder; ce qui lui importe, c'est de ne pas "trahir» les siens, de ne pas manquer à son "devoir». Tout effort de se préserver semble secondaire, la seule alternative plausible face à une vie de honte et de trahison est la mort sous la torture.

Nous sommes ici en présence d'une intériorisation de la perception héroïque du combattant, «l'homme ou la femme d'acier » qui se font détruire par la violence sans jamais tomber dans la déchéance explicite qu'elle entraîne. Il s'agit d'un déni ou d'un oubli de la réalité du corps martyrisé au nom d'un idéal héroïque et d'une métaphysique de la souffrance. Une perception magnifiée du combattant qui contraste avec la réalité imposée par les tortionnaires. Comme le dit si bien l'un d'eux, «si vous tous, vous aviez le droit de mourir, vous pourriez espérer devenir des héros, notre but est de vous transformer en vers de terre. $»^{27}$

Cet oubli de soi est manifeste dans l'expression testimoniale. Il s'accompagne même parfois du déni de toute une série d'images de soi qui ne seraient pas en conformité avec le modèle du discours du parti. Il s'agit chaque fois de promouvoir l'image d'un groupe de prisonnières «idéales ». Nous avons vu que les déportées à titre préventif constituaient une grande partie des prisonnières tout comme les combattantes de l'armée démocratique. Or, ces deux catégories ont pendant très longtemps fait l'objet d'une autocensure dans l'écriture testimoniale; les récits des femmes dont nous disposons sont plutôt condescendants à l'égard des déportées dites préventives, originaires des zones rurales et moins instruites que l'élite communiste urbaine. Et jusqu'aux années 90 , les témoignages publiés passent sous silence l'engagement militaire des combattantes. L'image féminine de la mère «injustement persécutée » doit prévaloir, l'opinion publique même de gauche a pendant longtemps du mal à accepter la présence des femmes de l'Armée démocratique, leur engagement est perçu comme une transgression de leur rôle social traditionnel.

Nous constatons alors que la mémoire testimoniale évolue beaucoup, et passe de l'évocation de l'exil et de l'expérience de la résistance dans les premiers textes des années 70, à l'évocation de la prison dans les années 80 pour oser enfin parler de la guerre civile et des femmes qui ont combattu dans l'Armée démocratique à partir des années 90. Cette expression mémorielle en trois temps, perceptible dans un moindre degré dans les témoignages des hommes, continue à laisser de côté la périphérie. Pour les prisons mixtes de province, nous connaissons très peu de choses, même si elles ont fonctionné jusqu'en 1952 et ont connu plus d'exécutions que les prisons d'Athènes. Finalement, la mémoire dominante a été celle d'une élite communiste, constituée des cadres du parti. La mémoire des signataires de déclarations de repentir est encore autocensurée, même si les signataires ne sont plus unanimement condamnés et que l'on commence à se les représenter en êtres humains qui ont dû négocier leur survie physique en sacrifiant un peu de leur idéal moral. 


\section{Conclusion}

$\mathrm{Au}$ moment de conclure, il nous semble intéressant de dégager certains aspects spécifiques à la réclusion des femmes. Habituées à endurer en silence, plus disciplinées, mais moins formées sur le plan politique, les femmes signent moins la déclaration de repentir que les hommes. Pourtant, elles sont plus vulnérables, et doivent endurer des sévices sexuels et du chantage relatif à la garde de leurs enfants. Pour expliquer leur sens de l'engagement, nous avons essayé de montrer à la fois leur adhésion au projet sociétal de l'EAM, leur quête de reconnaissance sociale, mais aussi leur constance et loyauté idéologiques. Pour leurs enfants, l'expérience de la prison reste un moment traumatique; des travaux récents montrent bien l'incidence de la réclusion dans leur vie d'adulte, tandis que les récits des prisonniers politiques nous décrivent avec force détails des situations cornéliennes où les mères doivent choisir entre l'amour de leurs enfants et la loyauté à leur parti. Enfin, et c'est peut-être là un des intérêts majeurs de ces récits, il existe une réelle évolution dans l'expression de la subjectivité, une réhabilitation du ressenti individuel face à l'expérience collective. Une distanciation de la transmission mémorielle fusionnelle, qui marque aussi l'avènement d'une nouvelle échelle de valeurs. La vision monolithique du Parti est supplantée par une approche critique, qui laisse plus de place au sujet; des témoignages où l'on s'interroge davantage sur ses propres choix, ses erreurs du passé, son sens de l'engagement.

La «culture de la terreur" ${ }^{28}$, longtemps déterminante dans la formation de la métamémoire ${ }^{29}$ de la guerre civile semble ainsi prête à céder le pas à une meilleure gestion de ses propres blessures et en même temps à une reconnaissance de la souffrance ${ }^{30}$ de l'Autre. Même si les communautés de mémoire (moins quand elles ont un ancrage empirique, une expérience propre du passé, et davantage quand elles sont fantasmatiques, sans vécu commun) ont toujours tendance à construire une version " officielle» du passé historique, l'expression testimoniale semble de plus en plus capable de se démarquer du discours dominant en son sein. Elle nous invite alors à réfléchir sur l'interaction entre transmission mémorielle des acteurs et influences métamémorielles du contexte interprétatif (discours historiques ou ethnologiques, enjeux idéologiques, actualité journalistique).

BIBLIOGRAPHIE

Ouvrages généraux en français, anglais et macédonien

Acheimastos, Myron, (non daté), L'idéologie communiste comme facteur de résistance dans l'île d'exil Makronissos pendant la période juillet 1948-mai 1950, DEA de sociologie générale, Université de Paris VII, pp. 3 et 10.

Alivizatos, Nikos, (1970), Les institutions politiques de la Grèce à travers les crises, 1922-1974, Paris : LGDJ. 
Avdela, Efi, (1998), «Quand classe et genre s'opposent : le cas de l'historiographie grecque contemporaine » in Sohn Anne-Marie - Thelamon Françoise (dir.) L'histoire sans les femmes est-elle possible?, Paris : Perrin.

Avdela, Efi, (2003), «L'histoire des femmes au sein de l'historiographie grecque contemporaine » in Bock Gisela - Cova Anne (dir.) Écrire l'histoire des femmes en Europe du Sud, XIX ${ }^{e}-X^{e}$ siècles/ Writing Women's History in Southern Europe: 19th-20th Centuries, Lisbonne : CELTA Editora.

Birtek, Faruk - Dragonas, Thalia, (2004), Citizenship and the Nation State: Greece and Turkey, et notamment les articles de Mouzelis Nicos et Pagoulatos George "Civil society and citizenship in post-war Greece", (pp. 87-103) et de Avdela Efi "Between Duties and Rights: Gender and Citizenship in Greece, 1864-1952”, (pp. 117-143), Londres: Routledge.

Boeschoten, Riki van, (1991), From armatolik to the people's rule. Investigation into the collective memory of rural Greece. 1750-1949, Amsterdam : Hakkert.

Dalegre, Joëlle, (2005), La Grèce depuis 1940, Paris : L'Harmattan,.

Dalegre, Joëlle, Andartika. Chants de la résistance grecque, L'Harmattan, Paris, 2008.

Dalianis-Karambatzakis, Mando, (1994), Children Turmoil during the greek civil war 1946-1949: Today's Adults. A longitudinal study on children confined with their mothers in prison, Stocholm : Karolinska Institutet.

Fourtouni, Eleni (ed.), (1986), Greek women in the resistance: Journals, Oral histories, New Haven: Lake View Printing.

Gritsi-Milliex, Tatiana, (1992), De l'autre rive du temps, Balzac, Édition du Griot éditeur.

Hart, Janet, (1996), New Voices in the Nation: Women and the Greek Resistance, 1941-1964, Ithaca: Cornell University Press.

Kaminis, Georgios, (1993), La transition constitutionnelle en Grèce et en Espagne, Paris : LGDJ, coll. «Bibliothèque constitutionnelle et science politique ».

Levita, David J. de, (2000), “Child Psychotherapy as an Instrument in Cultural Research: Treating War-Traumatized Children in the Former Yougoslavia" in Robben, Antonius \& Suarez-Orozco, Marcelo, Cultures under Siege. Collective Violence and Trauma, Cambridge: Cambridge University Press, (pp. 131-154).

Martinova-Buckova, Fana, (1998), I nie sme detsa na majkata zemja..., Skopje, Matitsa.

Mazower, Mark, (1997), "Policing the Anti-Communist State in Greece, 1922-1974", in Mazower, M. (éd.), The policing of politics in the twentieth century, Providence and Oxford: Berghahn, (pp. 129-150).

Milliex, Roger, (1945), Les Universitaires et Intellectuels de Grèce au service de la Résistance, Paris. Milliex, Roger, (1979), Hommage à la Grèce 1940-1944. Textes et témoignages français recueillis et présentés par Roger Milliex, Institut français d'Athènes.

Missios, Chronis, (1991), Toi au moins, tu es mort avant, La Tour d'Aignes.

Papadopoulou, Ourania, (1997), Femmes et politiques dans les années de répression en Grèce. Le cas de la PEOPEF (1948-1954), Mémoire de DEA de Sciences Sociales, Paris : EHESS.

Papathanassiou, Ioanna, (1988), Contribution à l'histoire du parti communiste grec, Thèse de doctorat, Nanterre : Université de Paris X. 
Panagiotopoulos, Georges-Panayotis, (1994), Réclusion et idéologie, une sociologie politique de la réclusion à partir de l'expérience des communistes grecs, Mémoire de DEA de sociologie, sous la direction de Michel Wieviorka, Paris : EHESS.

Panagiotopoulos, Georges-Panayotis, (2000), La morale communiste, Étude sur le rapport éthique dans les partis communistes d'Europe : URSS, France, Grèce, Thèse de doctorat sous la direction de M. le Professeur, Étienne Balibar, Nanterre : Université de Paris X.

Voglis, Polyméris, (2002), Becoming a subject: Political Prisonners during the Greek Civil War, 1945-1950, Oxford: Berhahn Books.

Dokumenti za ucestvoto na makedonskiot narod od egeskjiot del na Makedonija vo gra ganskata vojna vo Grcija 1945 godina [Documents relatifs à la participation du peuple macédonien de la Macédoine de l'Égée à la guerre civile en Grèce 1945], (1973), Préface, annotations des documents et table des matières disponibles en français, Skopje : Ahiv na Makedonija. Dans la même série, il y a des documents relatifs à la période 46-48.

Volkan, Vamik \& Itzkowitz, Norman, (2000). "Modern Greek and Turkish Identities and the Psychodynamics of Greek-Turkish Relations" in Robben, Antonius \& Suarez-Orozco, Marcelo, Cultures under Siege. Collective Violence and Trauma, Cambridge: Cambridge University Press, (pp. 131-154).

\section{Ouvrages en grec, translittérés en caractères latins.}

Abatzopoulou, Franggiski, (2000), « Istories zois. I vasanos kai i afigisi » in I grafi kai i vasanos. Zitimata logotehnikis anaparastasis, pp. 338-362, Athènes, Ekdoseis Pataki.

Abatzopoulou, Franggiski, (2000), « Istoria kai mythoplasia : oi autoviografikes afigiseis polemou » in I grafi kai i vasanos. Zitimata logotehnikis anaparastasis, Athènes : Ekdoseis Pataki, (pp. 338-362).

Apostolopoulou, Nathalia, (1985), Den doulono ...Den apografo, Athènes : Synchroni Epohi. Asdrahas, Spyros, (2000) «I martyria tis Virgos Vassileiou », Arxeiotaxio, vol. 2, Athènes, (pp. 128-136).

Avdela, Efi, (2002). « Dia logous timis ». Via, sunaisthimata kai axies sti metemfuliaki Ellada, Athènes : Nefeli.

Avdela, Efi, «I Istoria tou fulou stin Ellada: apo ti diatarahi stin ensomatosi ? », communication au colloque To fulo, topos sunantisis ton epistimon, enas protos ellinikos apologismos, tenu à l'Université de l'Égée (Mytilène) le 11 et le 12 octobre 2003.

Avdela, Efi, Psara, Angélica, (1997) «Eisagogi : Xanagrafontas to parelthon. Sunchrones diadromes tis istorias ton gunaikon» in Avdela Efi, Psara Angélica, (dir.) Siopires istories. Gunaikes kai fulo stin Istoriki Afigisi. Athènes : Alexandreia, (pp. 15-119).

Bærentzen, Lars, (2002), « To paidomazoma kai oi paidoupoleis tis vassilissas », (pp. 137-164) in Lars Baærentzen, Giannis Iatridis et Ole Smith, (dir.) Meletes gia ton emfulio polemo 1945-1949, Athènes : Olkos (pp. 137-164).

Beikos, Georgoulas, (1976), EAM kai laiki autodioikisi, Thessaloniki.

Beikos, Georgoulas, (1979), I laiki exousia stin eleftheri Ellada, Athènes : Themelio

Boeschoten, Riki van, (1997), Anapoda chronia. Syllogiki mnimi kai istoria sto Ziaka Grevenon, Athènes : Plethron.

Boeschoten, Riki van, Perasame polles bores koritsi mou, Athènes : Plethron, 1998. 
Boeschoten, Riki van, Vervenioti Tasoula, Voutyra Eutychia, Dalkavoukis Vassilis et Bada Konstantina, (dir.), (2008), Mnimes kai lithi tou ellinikou emfyliou polemou, Salonique : Epikentro. Boutzouvi-Bania, Aleka, (1993), « Proseggisi sto odoiporiko mias gunaikas : Diamando TsiakaGritzona », Dini, féministiko périodiko n 6,(pp. 196-229).

Boutzouvi-Bania, Aleka, “"To anthropino hreos” exei fulo: i periptosi tis Evaggelias Samara», Dini, féministiko périodiko 8 (1995-1996).

Falianga-Papanikolaou, Eleni, (1980), à partir des textes de Tasia Mamida Theodorakou, Stis manes mas. Marturies apo tin fulaki, Athènes : Ekdoseis apo fotia kai apo sidero.

Falianga-Papanikolaou, Eleni, (1982), I Angelo, Athènes : Ekdoseis apo fotia kai apo sidero. Falianga-Papanikolaou, Eleni, (1983) Xylo i Peitho, Athènes : Ekdoseis apo fotia kai apo sidero. Falianga - Papanikolaou, Eleni (1985), An pas sto Makronisi, Athènes : Ekdoseis apo fotia kai apo sidero.

Gagoulias, Yorgos, (2005), O emfylios polemos kai to paidomazoma, Athènes : Iolkos.

Georgopoulou-Ioannidou, Mairi, (1981), Pikri epohi, Kavala.

Hatzi, Iro, (1999), ESEIS, EMEIS, eseis, Athènes : Dorikos.

Imvrioti, Rosa, (1985), Dokoumenta apo to arheio tis Pozas Imvrioti, [Documents tirés des archives de Rosa Imvrioiti], (mai 1946), vol. 1, Athènes : Omospondia Gynaikon Elladas.

Karagiorgi, Maria, (1989), Mehri tin apodrasi, Athènes : Fytrakis.

Karagiorgi, Maria, (2001), Apo mia spitha xekinise, Athènes : Proskinio.

Karagiorgi, Maria, (2005), Perimenontas tin eirini, Athènes : Proskinio-Aggelos Sidératos.

Karanika, Soula, (1984), Gia mia nea zoi, Athènes : Ekdoseis Synchroni Epohi.

Karra, Maria, (1995), Emeis oi ap'exo. PEOPEF, 1948-1954. Mia « mikri epopoia », Athènes : Kostarakis.

Kotsaki, Margarita, (1987), Mia zoi gemati agones, Athènes : Ekdoseis Synchroni Epohi.

Koundouros, Roussos, (1978) I asfaleia tou kathestotos, Politikoi kratoumenoi, Ektopiseis kai Taxeis stin Ellada, 1924-1974, Athènes : Kastaniotis.

Kounio-Amarilio, Erika, (1995), Peninta hronia meta... Anamniseis mias salonikiotissas Evraias, témoignage recueilli par Franggiski Abatzopoulou, Salonique : Paratiritis.

Lagani, Eirini, (1996), To “paidomazoma” kai oi ellino-yugoslavikes scheseis (1949-1953), Athènes : I. Sidéris.

Lambropoulou, Dimitra, (1999), Grafontas apo tin fulaki, Athènes : Nefeli.

Lazarou, Fofi, (2006), «I heirafetisi ton gunaikon stin antistasi» in Stélios Kouloglou Marturies gia ton emfulio kai tin elliniki aristera, Athènes : Vivliopoleio tis Estias.

Manolkidou-Vetta, Fani, (1997), Tha se leme Ismini. Oi anamniseis einai megaluteres apo ti zoi, Athènes : Philistor.

Mara-Mihalakea, Dimitra, (1995), Gynaikeies fylakes Averof. Athina 12-13 apriliou 1949, Athènes : Elegeia, Agra.

Mastora-Psaroyanni, Olga, (1995), Sto dromo tou xreous, Athènes : Ekdoseis Synchroni Epoxi. Mastroleon-Zerva, Marigoula, Exoristes, (1986), Athènes : Synchroni Epohi.

Missios, Chronis, (1988), « Hamogela, re... Ti sous zitane? ", Athènes : Éditions grammata. 
Papadouka, Olympia, (2006), Gynaikeies Fulakes Averof, Athènes : Diogénis.

Papataxiarhis, Efthymios - Paradellis, Théodoros, (dir.), (1992), Taftotites kai fulo sti sunchroni Ellada. Anthropologikes proseggisei, Athènes : Kastaniotis.

Papataxiarhis, Efthymios, (1997), « To fulo stin anthropologia (kai tin istoriografia) : orismenes gnostikes kai methodologikes proektaseis», Mnimon 19, (pp. 201-210).

Sakellariou, Charis, (1984), I paideia stin Antistasi, Athènes : Synchroni Epochi.

Sakellariou, Charis, (1995), To theatro tis antistasis, Athènes : Synchroni Epochi.

Samiou, Dimitra, (1989), « Staseis kai antistaseis apenanti sto dikaioma psifou ton gunaikon mesa apo ton Tupo : 1919-1952 », Dini féministiko périodiko 4, (pp. 29-35).

Samiou, Dimitra, (1989), « Ta politika dikaiomata ton Ellinidon (1864-1952) », Mnimon 12, (pp. 161-172).

Servos, Dimitris, (2004), To paidomazoma kai poioi fovountai tin alitheia, Athènes : Synchroni Epohi. Sideri, Maria, (1981), Dekatessera hronia, Martyria, Athènes : ekdoseis Ermis.

Sideri, Maria, (1992), Hronia Odynis, Athènes : Mohlos.

Skarpeti-Tsali, Eleni, (1985), Stamateiste, sevasteite tous anthropous, Athènes.

Skouteri-Didaskalou, Nora, (1991), Anthropologika gia to gunaikeio zitima, Athènes : O Politis.

Stefanou, Pagona, (1998) Ton afanon. Duo tetradia, duo tetraeties, Athènes : Thémélio.

Staveri, Ourania, (2006), To martyriko trigono ton exoriston gunaikon, Hios, Trikeri, Makronissi, Athènes : Paraskinio, (pp. 100-101).

Tsakiri, Sasa, (1985), Kleisti strofi, Athènes : Kastaniotis.

Tsakiri Sasa, (1996), Dido Sotiriou. Apo ton kipo tis Edem sto kamini tou aiona, Athènes : Kedros.

Tsouparopoulos, Thanasis, (1989), Oi laokratikoi thesmoi tis Ethnikis Antistasis, Athènes : Glaros. Vardinoyannis, Vardis et Aronis, Panayotis, (1996), Oi misoi sta sidera, Athènes : Philistor.

Vervenioti, Tassoula, (1993), «I thesmothetisi tou dikaiomatos tis psifou ton gunaikon apo to elliniko antistasiako kinima (1941-1944) », Dini, féministiko périodiko, vol. 6, (pp. 180-195).

Vervenioti, Tassoula, (1994), I gynaika tis antistasis. I eisodos ton gynaikon stin politiki, Athènes : Odysseas.

Vervenioti, Tassoula, (1999), « To dilimma kai to timima ton gunaikon tis Antistasis », Ta Istorika 31.

Vervenioti, Tassoula, (2000) « Makronissi, martyria kai martyries gunaikon » in Istoriko topio kai istoriki mnimi. To paradeigma tis Makronissou, Praktika Epistimonikis Synantisis, Athènes 6-7 mars 1998, Athènes : Philistor, (pp. 103-114).

Vervenioti, Tassoula, (2003), To diplo vivlio, Athènes : Vivliorama.

Victoria, Théodorou (dir.), (1976 $1^{\text {ere }}$ édition), Stratopeda gunaikon, ennea thammena tetradia me afigiseis kratoumenon gunaikon sta stratopeda Hiou, Trikeri, Makronissou, sta hronia tou emfyliou polemou, 1947-1951, réédité par l'Association des Femmes Déportées et Prisonnières Politiques en 2002, Athènes : Alfeios.

Vlassi Theodorikakou, Alexandra, (1997), Oi mnimes menoun zontanes. Oi anamniseis mias 17xronis antartopoulas tou DES, Athènes : Ekdoseis Entos. 
Yannopoulou-Trianti, Alexandra, (1990), Orthies sti thyella, Gynaikeies Fylakes Patras, 1948-1952, Athènes : Vivliopoleio Xrusafi Panezi.

\section{NOTES}

1. Cf. Polyméris Voglis, L'expérience de la prison et de la déportation, les prisonniers politiques

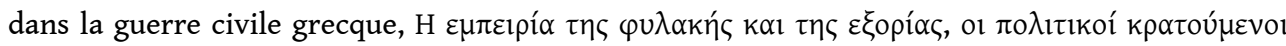

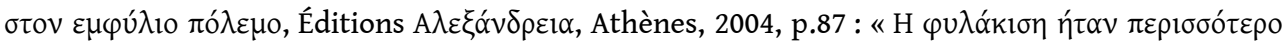

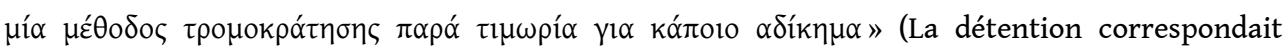
davantage à une méthode d'intimidation qu'à une sanction d'un quelconque délit) et pp. 81-105.

2. Cf. Nikos Alivizatos, Les institutions politiques de la Grèce à travers les crises, 1922-1974, LGDJ, Paris, 1970 et également G.Kaminis, La transition constitutionnelle en Grèce et en Espagne, coll. «Bibliothèque constitutionnelle et science politique ", $t$ 16, Paris, LGDJ, 1993. Dans cet ouvrage, l'auteur traite des «séquelles de la guerre civile » qui se sont traduites en Grèce et en Espagne par l'exclusion des communistes et des républicains respectivement.

3. Riki Van Boeschoten parle d'habitus de l'EAM dans le sens que Bourdieu lui accorde en tant que structure structurante. Le nouvel habitus de l'EAM, première expression d'esprit civique en Grèce moderne, vient en quelque sorte supplanter les anciens habitus de la tradition des Klephtes et du monde rural, tout en intégrant certains éléments de ces structures. L'habitus de l'EAM correspond à des valeurs et des comportements qui sont aux antipodes de l'habitus épique de la tradition klephte et qui rompent avec l'archaïsme social de l'habitus rural : l'EAM met en avant des valeurs collectives au détriment de l'idéal héroïque individualiste, réfute l'usage de la violence, prône les pratiques de réconciliation et d'entraide, institue la démocratie directe, fait de l'accès à l'éducation et de la gratuité des soins une priorité immédiate, applique l'égalité des sexes, propose de nouveaux rôles aux femmes et aux jeunes, en deux mots, il œuvre pour une démocratisation de la société grecque. Auprès des masses populaires, notamment en zone rurale, son action est généralement considérée comme une alternative politique d'autant plus plausible que la réalité immédiate des pratiques inaugurées, discrédite, encore plus, un pouvoir central, perçu comme collaborationniste ou absent. Pour l'anthropologue néerlandaise, l'habitus des klephtes, armatoles et autres groupes armés entretenant des rapports ambivalents avec le pouvoir central tantôt au service de ce dernier et tantôt en révolte contre lui, est porteur d'un premier ensemble de valeurs compatibles avec le mode de vie traditionnel des communautés transhumantes, où un code d'honneur archaïque fondé sur l'héroïsme individuel, la parole donnée et la légitimation de la violence et de la méfiance vis-à-vis de l'autorité étatique, régit tous les rapports sociaux. L'habitus rural déterminé par des règles de vie en communauté solidaire est porteur quant à lui des valeurs de réciprocité, d'entraide et de tolérance qui s'opposent à l'agressivité du modèle du Klephte. Pour une étude détaillée de ces quatre habitus,

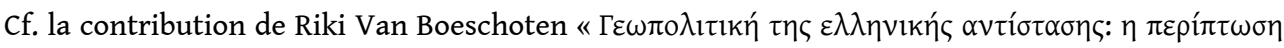

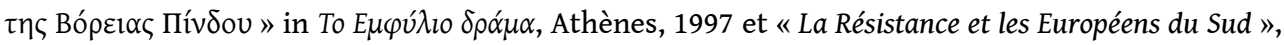
Actes du colloque d'Aix-en-Provence, 20-22 mars 1997, Paris, L'Harmattan, 1999. Pour le modèle rural français, «La France rurale et la Résistance », in Sarah Fishman, Laura Lee Downs, Ioannis Sinanoglou, Leonard V. Smith et Robert Zaretsky (sous la direction de) et « Le monde rural face au maquis ", in François Marcot (sous la direction de), La Résistance et les Français. Lutte armée et maquis, actes du colloque de Besançon, 15-17 juin 1995, Besançon, Annales littéraires de l'Université de Franche-Comté, 1996. Sur le code d'honneur de la société grecque Cf. Riki Van Boeschoten, From armatolik to the people's rule. Investigation into the collective memory of rural Greece. 1750-1949, Hakkert, Amsterdam, 1991. 
4. Sur les « orphelinats de la reine ", le traumatisme de la séparation et les manques affectifs de ces enfants placés en institution et dressés contre leurs parents, jugés «traîtres ", il existe quelques travaux de référence menés par Riki Van Boeschoten. Voir, la contribution de Riki Van Boeschoten (en grec) «Mémoires, traumatismes et métamémoire: la "levée" d'enfants et

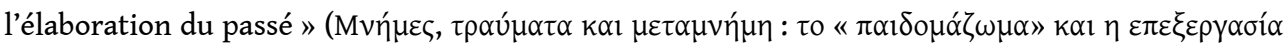

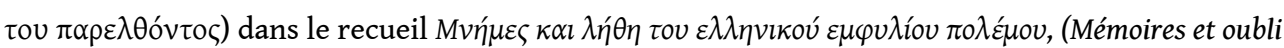
de la guerre civile grecque) sous la direction de Riki Van Boeschoten, Tasoula Vervenioti, Eutychia

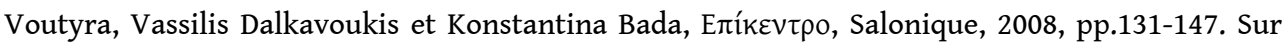
les conditions de détention des enfants en prison et l'impact psychologique de cette expérience, voir les travaux de Mando Dalianis-Karambatzakis, Children Turmoil during the greek civil war 1946-1949: Today's Adults. A longitudinal study on children confined with their mothers in prison, Karolinska Institutet, Stocholm, 1994.

5. Pour T. Vervenioti la catégorie des personnes exilées à titre préventif, comportait essentiellement des femmes qui avaient eu un engagement réel lors du conflit et n'impliquait pas

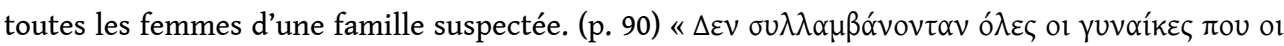

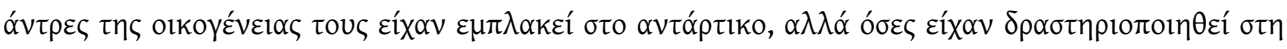

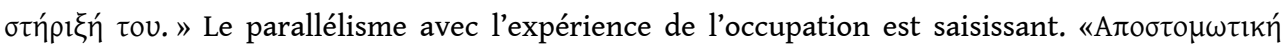

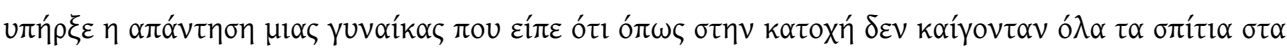

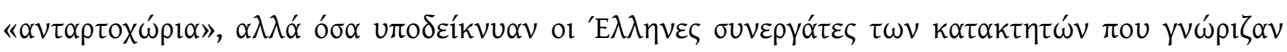

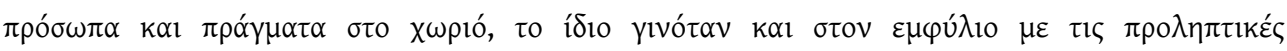

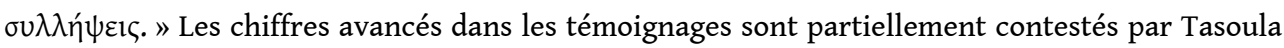
Vervenioti, dans sa contribution «Mémoires et oublis des archives et des témoignages pour la guerre civile grecque. Athènes et la province, la direction et les membres » (pp.81-147- en grec) in Van Boeschoten Riki, Vervenioti Tasoula, Voutyra Eutychia, Dalkavoukis Vassilis et Bada Konstantina, (sous la direction de) Mnimes kai lithi tou ellinikou emfyliou polemou Salonique, Epikentro, 2008. L'historienne grecque compare les chiffres qui ressortent de son travail sur les témoignages à ceux des Archives de la Croix rouge Internationale et montre une double tendance dans la mémoire testimoniale «officielle»: l'oubli quasi-systématique des femmes d'origine rurale, peu instruites ou mal positionnées dans la hiérarchie du parti et la condescendance à l'égard des signataires des déclarations de repentir et les personnes exilées à titre préventif.

6. Citons à titre d'exemple, le cas de deux nouveaux-nés, dont la mère est Macédonienne,

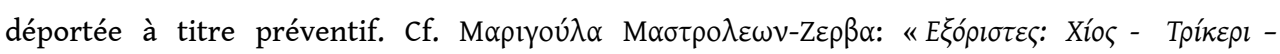

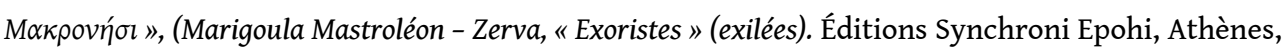
1986.

7. À signaler également le cas de Marika épouse de Bartziotas qui refuse de signer sous la torture

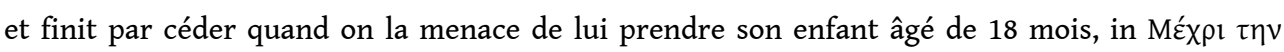

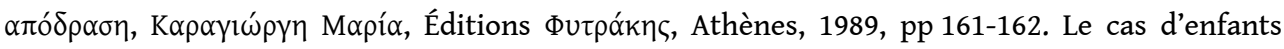
d'abord envoyés dans des camps de concentration ou des prisons avec leurs mères, pour en être séparés quelque temps après est magistralement traité dans le livre de Mando DalianisKarambatzakis, Children in Turmoil during the Greek Civil War 1946-49: Today's Adults, Karolinska

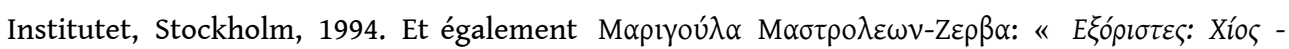

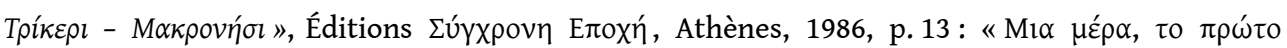

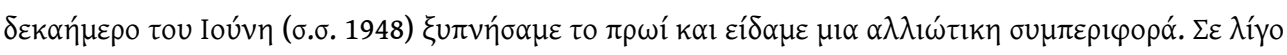

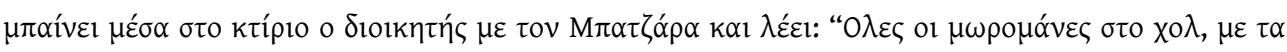

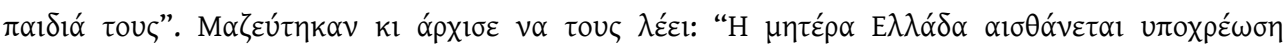

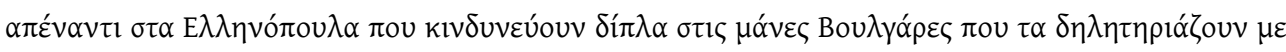

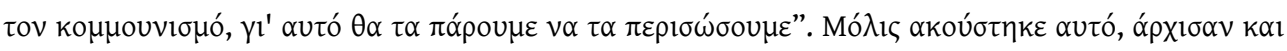

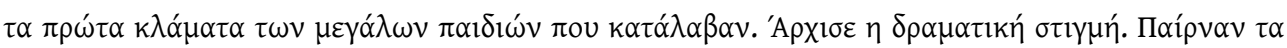




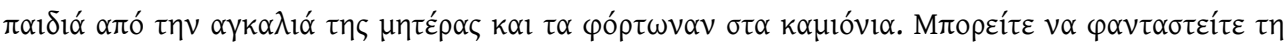

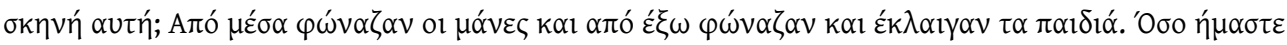

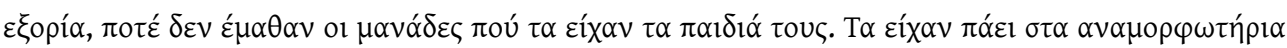

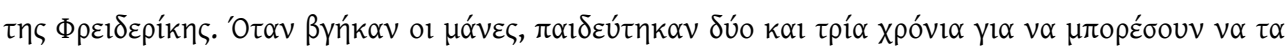

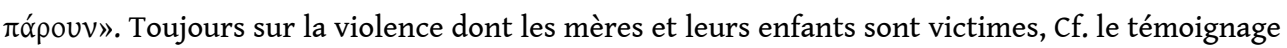

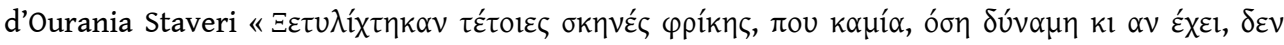

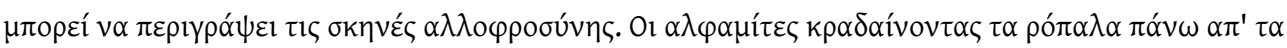

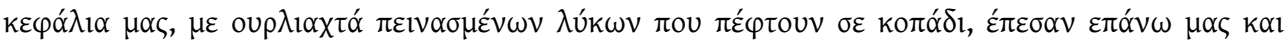

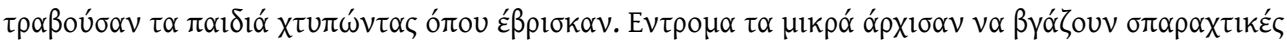

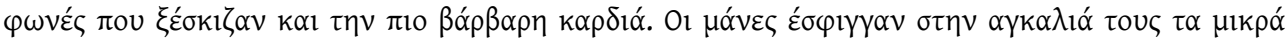

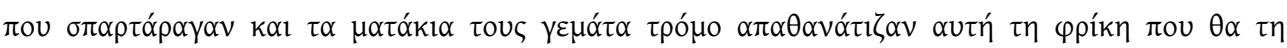

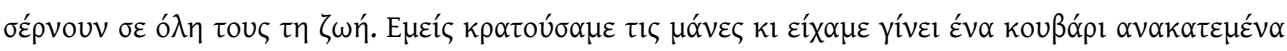

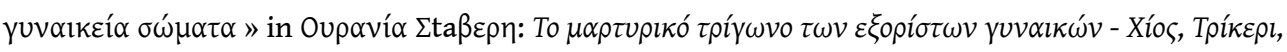

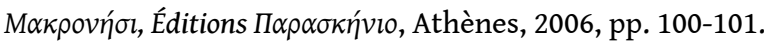

8. Des enfants qui meurent des maladies déjà citées ou qui subissent des peines d'emprisonnement en cellule d'isolement. Voir le cas, de Roula, âgée de neuf ans. Son histoire est citée dans Victoria Théodorou (sous la direction de) Stratopeda gunaikon, ennea thammena tetradia me afigiseis kratoumenon gunaikon sta stratopeda Hiou, Trikeri, Makronissou, sta hronia tou emfyliou polemou, 1947-1951, Athènes, 1976, réédité par l'Association des Femmes Déportées et Prisonnières Politiques en 2002 aux Éditions Alfeios.

9. Cf. La contribution de Riki Van Boeschoten (en grec) "Mémoires, traumatismes et

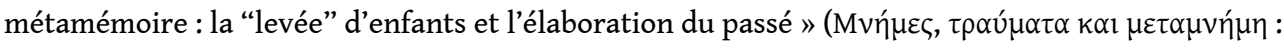

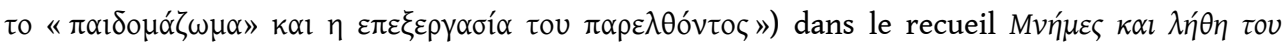

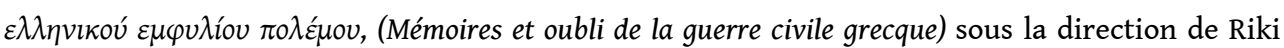
Van Boeschoten, Tasoula Vervenioti, Eutychia Voutyra, Vassilis Dalkavoukis et Konstantina Bada,

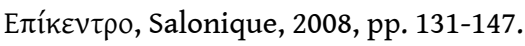

10. Cf. Mando Dalianis-Karambatzakis, ibidem, p. 18.

11. Cf. Ibid, p. 19.

12. Cf. G. Gagoulias, La "levée » d'enfants dans les temps orageux de la guerre civile grecque, To

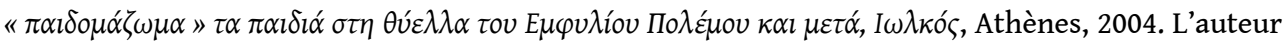
y présente les conditions d'accueil, les difficultés que les enfants et les enseignants ont dû affronter et le rôle de l'EVOP (Comité d'aide à l'enfant) dans l'intégration et l'instruction de ces enfants.

13. L'accueil en prison suit un rituel, où ceux qui arrivent sont pris en charge par les anciens; on leur propose un verre d'eau, un fruit (s'il y en a), une place où dormir ; c'est un processus de réaffirmation de la subjectivité dans le cadre d'une collectivité rassurante et solidaire.

14. P. Panayotopoulos ibidem p. 112-114.

15. Natalia Apostolopoulou, Coup de poing dans la pénombre, Synchroni Epochi, Athènes, 1984, (en grec), passim. Voir aussi l'ouvrage collectif sous la direction de Victoria Théodorou, Camps de femmes, Athènes, 1976 (en grec).

16. Marigoula Mastroleon-Zerva, Détenues à Youra, 1967, Kapopoulos, (en grec), pp. 43-45.

17. Olympia V. Papadouka, Prison de femmes Avéroff, Athènes, 1987, (en grec) pp. 23-35, ainsi que Marigoula Mastroleon-Zerva, Exilées- Chios, Trikeri, Makronissos, op.cit. pp. 87, 99.

18. On rencontre de temps en temps bien sûr des pratiques analogues dans des camps pour hommes, mais leur rareté ainsi que la difficulté que les auteurs des témoignages ont pour les conter est significative de leur caractère marginal.

19. M. Mastroléon-Zerva, Exilées..., op.cit. pp. 9-11.

20. M. Mastroléon-Zerva, Détenues à Youra, op.cit. pp. 31-32. 
21. Les thèses du PCG sur la question des femmes, sa guerre ouverte avec le mouvement féministe alternatif ainsi que l'appauvrissement de la problématique de la domination structurelle des femmes par les hommes au profit du réductionnisme économique le plus vulgaire sont des constantes dans son discours que l'on retrouve encore aujourd'hui. Voir à ce sujet, Aléca Papariga, Pour la libération de la femme, Synchroni Epochi, 1986, (en grec), pp. 35, 36, 40-48, 54, 57, 64-70, 78-83, 88, 91, 94-97, 103-109, 121-125.

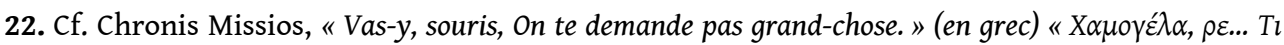

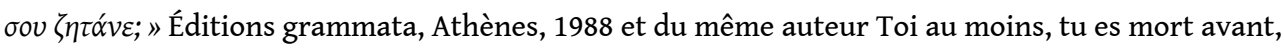
La Tour d'Aignes, 1991.

23. Myron Achéimastos, L'idéologie communiste comme facteur de résistance dans lîle d'exil Makronissos pendant la période juillet 1948-mai 1950, DEA de sociologie générale, Paris VII, non daté, pp. 3 et 10.

24. Le cas d'Eleni Skarpeti, détaillé dans l'ouvrage de Olumpia Papadouka, Prisons de femmes Avéroff, (en grec), Diogenis, Athènes, 2006 et cité dans le journal Kathimerini du 12/10/2008, est révélateur de la discrimination qui frappait les signataires, les dissidents et tous ceux qui se

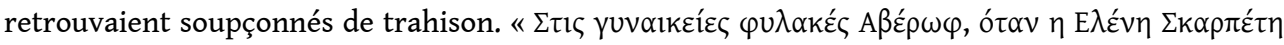

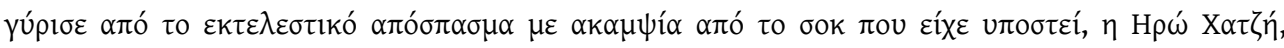

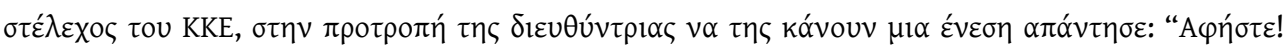

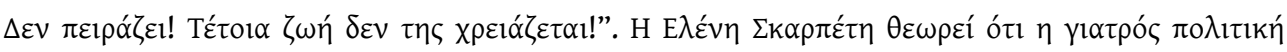

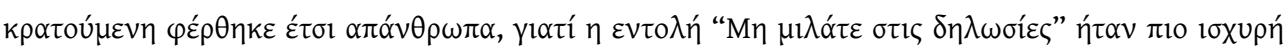

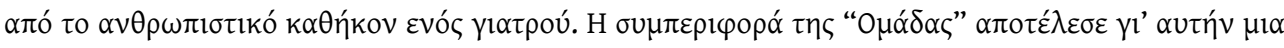

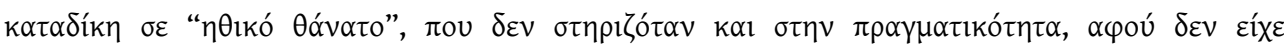

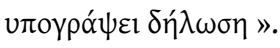

25. "Il faut penser à sa femme comme à une compagne de lutte». L'article dix du code de conduite en exil, élaboré par le PCG impose l'abstention sexuelle dans les îles où des femmes et des hommes étaient déportés et vivaient en mixité, pour éviter de choquer la population locale. Il faut dire que la propagande gouvernementale s'était beaucoup amusée à dénoncer le « libertinage » supposé des organisations de jeunesse ou du maquis.

26. Panayotopoulos, ibidem. p. 108.

27. Cf. Victoria Théodorou, Ibidem.

28. Michael Taussig, "Culture of Terror, Space of Death", in Joan Vincent (éd.) The anthropology of Politics, A Reader in Ethnography, Theory and Critique, Oxford, Blackwell, 2002, p. 173.

29. Terme employé par Marianne Hirsch pour parler de la mémoire des enfants qui ont survécu à l'Holocauste. Cf. Marianne Hirsch, Family Frames:Photography,Narrative and Postmemory, Harvard University Press, 1997.

30. La "levée » d'enfants jouerait dans ce sens le rôle d'un "traumatisme choisi ", "chosen trauma ». Cf. Vamik Volkan \& Norman Itzkowitz, "Modern Greek and Turkish Identities and the Psychodynamics of Greek-Turkish Relations" in Antonius ROBBEN \& Marcelo SUAREZ-OROZCO, (pp. 131-154), Cultures under Siege. Collective Violence and Trauma, Cambridge, Cambridge University Press, 2000. 


\section{RÉSUMÉS}

Les conditions et stratégies de survie des milliers de femmes condamnées ou déportées de la guerre civile grecque permettent d'établir une typologie.

Pendant la guerre civile grecque (1946-1949) des milliers de personnes sont emprisonnées, déportées dans des camps de concentration ou exilées dans des îles, une population hétéroclite d'hommes et de femmes, persécutés pour leurs idées, leurs origines ou même des liens de parenté avec les communistes. Dans la présente étude, nous nous intéresserons à un groupe lui-même peu homogène, les femmes et les enfants, prisonniers politiques ou déportés à titre préventif pendant le conflit. Après un bref rappel de la pratique de la déportation et une esquisse de typologie de la réclusion, nous étudierons les conditions de détention et les stratégies de survie des femmes, souvent emprisonnées avec leurs enfants, leur statut au sein des prisonniers politiques ainsi que le sens de l'engagement politique dans leur système de valeurs.

During the Greek Civil War (1946-1949) thousands of persons were imprisoned, deported to concentration camps or exiled on the islands, a heteroclite population of men and women, persecuted for their ideas, their origins or even their association with the Communists. In the present study, we are interested in a not particularly homogeneous group of women and children, political prisoners or deportees who had been detained for preventive reasons during the conflict. Following a brief summary of the practice of deportation and a typological sketch of the imprisonment, we shall study the detention conditions and the survival strategies of the women, often imprisoned with their children, their status vis-à-vis the political prisoners as well as the meaning of political commitment in their value system.

\section{INDEX}

motsclestr Yunanistan, Makronisos, Trikeri, Sakız, Yunan İç Savaşı (1946-1949)

motsclesmk ГРцИЈА, ХИос

Thèmes : Histoire

Index chronologique : guerre civile grecque (1946-1949)

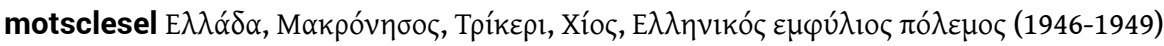

Mots-clés : Acronauplie, anticommunisme, femmes dans la guerre, Chios, EAM, prisonniers politiques, Makronissos, réclusion politique, Trikéri

Index géographique : Grèce, Makronissos, Trikéri, Chios

Keywords : anticommunism, women in war, political prisoners, Greece, Makronissos, Trikeri, Chios, History

\section{AUTEUR \\ CHRISTINA ALEXOPOULOS}

CC INALCO, Doctorante CREE-CEB EA 4513 